\title{
Asymptotic Portfolio Strategy Based on the CEV Model with General Utility Function
}

\author{
Yu Jia $\mathbb{D}^{1},{ }^{1}$ Liyun Su $\mathbb{D},{ }^{1}$ Yong He $\mathbb{D}^{2},{ }^{2}$ and Qi Huang ${ }^{3}$ \\ ${ }^{1}$ College of Science, Chongqing University of Technology, Chongqing 400054, China \\ ${ }^{2}$ School of Mathematics, Physics and Data Science, Chongqing University of Science and Technology, Chongqing 401331, China \\ ${ }^{3}$ School of Mechanical and Power Engineering, Chongqing University of Science and Technology, Chongqing 401331, China
}

Correspondence should be addressed to Yu Jia; 931571948@qq.com and Yong He; 402942832@qq.com

Received 29 June 2021; Revised 17 September 2021; Accepted 8 October 2021; Published 1 November 2021

Academic Editor: Eric Florentin

Copyright (@) $2021 \mathrm{Yu}$ Jia et al. This is an open access article distributed under the Creative Commons Attribution License, which permits unrestricted use, distribution, and reproduction in any medium, provided the original work is properly cited.

\begin{abstract}
The optimal investment problem is a hot field of financial risk control. The analytical solution of investment strategy can be obtained with the power function utility and exponential function utility when the stock price obeys the constant elasticity of variance (CEV) model. However, different investors have different risk preferences; it means that different investors have different utility functions. In this paper, we propose an asymptotic analysis method to obtain the asymptotic solution of investment strategy with the general utility function. The value function is expanded in the form of series, the expressions of the zero-order term and first-order term of the series expansion are derived, respectively, and the error between the asymptotic approximation and the optimal value function is calculated. Finally, the numerical examples provide comparative analysis between the analytical solution and the asymptotic solution to verify the effectiveness of the proposed method.
\end{abstract}

\section{Introduction}

Investment portfolio is a collection of stocks, bonds, financial derivatives, etc., held by investors or financial institutions, with the purpose of diversifying risks. Usually investment portfolio is composed of two financial assets in the market: one is risk-free assets (bank deposits, etc.) and the other is risky assets (stocks, etc.). Given time $t$ and initial assets including various known parameters, investors want to find optimal portfolio strategy to maximize the expected wealth utility at time $T$. Merton proposed the optimal portfolio problem in the case of continuous time in 1969, constructed the Hamilton-Jacobi-Bellman equation (HJB equation) satisfied by the objective value function by using the principle of dynamic programming, and then obtained the optimal investment strategy under the Black-Scholes (BS) model according to the homogeneity of the utility function. However, the volatility coefficient of BS stock price model is constant, which is inconsistent with most empirical data. Cox and Ross [1] proposed a constant elasticity of variance $(\mathrm{CEV})$ model to describe the stock price. The volatility is a power function of the stock price, which is no longer a constant. The CEV model is usually used to calculate the theoretical price, sensitivity, and implied volatility of options (Cox [2], Davydov [3], Detemple [4], Lo [5], Widdicks [6], and Yuen [7], and well explains the empirical deviations shown by the BS model, such as volatility smile. In the works of Beckers [8] and Emanuel and Macbeth [9], there are some theoretical arguments and empirical evidence to support the change of volatility with stock price and the negative elasticity coefficient. Gao [10] solved the optimal investment problem under the CEV model by using stochastic optimal control, Legendre transformation, and partial differential equation theories, and the analytical solutions are obtained under the power utility and exponential utility.

Different investors have different risk preferences, which means that they have different utility functions. Therefore, it is necessary to consider the investment problem with general utility functions. Ma [11] provides a method to solve the general utility function; the original problem is transformed into a dual problem through dual transformation; then, the 
upper and lower bounds of the value function are calculated by the Monte Carlo method. By continuously narrowing the range of the upper and lower bounds, the results meeting a certain accuracy are achieved, but this method needs good programming ability and long computing time. In this paper, we expand the value function in the form of Taylor series and calculate the approximate value function which contains zero-order and first-order terms; the higher-order terms (third-order and above) are neglected as the errors.

This paper considers that there are two kinds of financial assets in the financial market: one is risk-free asset (bank deposit) and the other is risk asset (stock). Based on the principle of dynamic programming, the HJB equation of the value function under the general utility function is established, and the first three terms of the Taylor series are obtained. Finally, we compare the error between the analytical solution and the asymptotic solution under the power function utility and the exponential function utility in the numerical part.

\section{Models and Theorems}

$B_{t}$ represents the price of risk-free assets (bank accounts) at time $t$, and the equation is given by

$$
\mathrm{d} B_{\mathrm{t}}=r B_{t} \mathrm{~d} t
$$

where $r$ is a risk-free interest rate and $S_{t}$ represents the price of risky assets at time $t$, which is described by the CEV model (Lo et al. [5] and Yuen et al. [7]):

$$
\frac{\mathrm{d} S_{t}}{S_{t}}=\mu \mathrm{d} t+k S_{t}^{\beta} \mathrm{d} W_{t}
$$

where $\mu$ is the expected instantaneous rate of return of the stock and satisfies the condition $\mu>r, k S_{t}^{\beta}$ is the instantaneous volatility of stock price, and $\beta$ is an elastic parameter that satisfies $\beta<0 .\left\{W_{t}: t \geq 0\right\}$ is a standard Brownian motion in the complete probability space $(\omega, \mathscr{F}, P)$ and $P$ is the probability. $\mathscr{F}=\mathscr{F}_{t}$ is the right continuous filter in this space, which represents the information structure generated by Brownian motion.

Let $X_{t}$ represent the wealth process, $t \in[0, T], \pi_{t}$ represent the amount of wealth invested in risk assets, and $X_{t}-$ $\pi_{t}$ represent the amount of wealth invested in risk-free assets. So, we have

$$
\mathrm{d} X_{t}=\pi_{\mathrm{t}} \frac{\mathrm{d} S_{t}}{S_{t}}+\left(X_{t}-\pi_{\mathrm{t}}\right) \frac{\mathrm{d} B_{t}}{B_{t}}, \quad X_{0}=x,
$$

where $x$ is the initial wealth value. Substituting (1) and (2) into (3), the wealth process obeys the following equation:

$$
\mathrm{d} X_{t}=\left[\left(\pi_{\mathrm{t}}(\mu-r)+r X_{t}\right)\right] \mathrm{d} t+\pi_{\mathrm{t}} k S_{\mathrm{t}}^{\beta} \mathrm{d} W_{t}, \quad X_{0}=x .
$$

Let $\mathscr{A}$ represent the admissible strategy set, and we define the value function $J(t, x, s)$ as follows:

$$
J(t, x, s):=\operatorname{ess}_{\sup } E\left[U_{T}\left(X_{T}^{\pi}\right) \mid X_{t}^{\pi}=x, s_{t}=s\right],
$$

under the boundary condition $J(T, x, s)=U_{T}(x)$.

Using the dynamic programming principle, the value function $J$ satisfies the following Hamilton-Jacobi-Bellman (HJB) equation:

$$
J_{t}+r x J_{x}+\mu s J_{s}+\frac{1}{2} k^{2} s^{2 \beta+2} J_{s s}+\max _{\pi}\left((\mu-r) \pi+\frac{1}{2} \pi^{2} k^{2} s^{2 \beta} J_{x x}+\pi k^{2} s^{2 \beta+1} J_{x s}\right)=0
$$

The optimal strategy $\pi_{t}^{*}$ is obtained according to the firstorder maximization condition of (6):

$$
\pi_{t}^{*}=-\frac{(\mu-r) J_{x}+k^{2} s^{2 \beta+1} J_{x s}}{k^{2} s^{2 \beta} J_{x x}} .
$$

Substituting formula (7) into the partial differential equation, we obtain

$$
J_{t}+r x J_{x}+\mu s J_{s}+\frac{1}{2} k^{2} s^{2 \beta+2} J_{s s}-\frac{\left[(\mu-r) J_{x}+k^{2} s^{2 \beta+1} J_{x s}\right]^{2}}{2 k^{2} s^{2 \beta} J_{x x}}=0 .
$$

We consider that the asymptotic solution is related to time $t$ and is expanded with the form of Taylor series. Theoretically, the higher the order of Taylor series, the smaller the error between the asymptotic solution and the real value function. However, it needs lots of calculations to obtain the higherorder terms. Therefore, in this paper, the second order and above are looked as errors. It is worth noting that, in [12], the difference between the subsolutions $J(t, x, y)$ and the optimal solution $\bar{J}(t, x, y)$ is in the second-order term, that is, the error term. A probabilistic argument using martingale inequalities will show that the value function lies between the constructed sub- and super-solutions.

Theorem 1. Let $\widehat{J}(t, x, s)$ be the value function of formula (5) and $U_{T}(x)$ be the terminal utility function, and we define

$$
\widehat{J}(t, x, s)=U_{T}(x)+(T-t) \frac{r x U_{T}^{\prime}(x)-\left(1 /\left(2 k^{2} s^{2 \beta}\right)\right)(\mu-r)^{2} U_{T}^{\prime}(x)^{2}}{U_{T}^{\prime \prime}(x)},
$$

satisfy $|J(t, x, s)-\widehat{J}(t, x, s)| \leq c_{2}(T-t)^{2}$, for all $\quad(x, s) \in$ $(0, \infty) \times(\infty, 0)$.

Proof. We expand the value function in the terms of $(T-t)$ as follows: 
$J(t, x, s):=J^{(0)}(x, s)+(T-t) J^{(1)}(x, s)+(T-t)^{2} J^{(2)}(x, s)$.

In order to make our first-order approximation consistent with the value function at the terminal time $T$, we assume the terminal condition of the value function as the value of $J^{(0)}$.
Namely, note that $J(t, x, s)$ satisfies the boundary condition $J(T, x, s)=U_{T}(x)$, so we have

$$
J^{(0)}(x, s):=U_{T}(x), \quad \text { for all }(x, s) \in(0, \infty) \times(\infty, 0) .
$$

Substituting $J^{(0)}(x, s)$ into (8), we obtain

$$
\begin{aligned}
& -U^{(1)}-2(T-t) U^{(2)}+r x\left[U_{x}^{(0)}+(T-t) U_{x}^{(1)}+(T-t)^{2} U_{x}^{(2)}\right]+\mu s\left[U_{s}^{(0)}+(T-t) U_{s}^{(1)}+(T-t)^{2} U_{s}^{(2)}\right] \\
& +\frac{k^{2} s^{2 \beta+2}}{2}\left[U_{s s}^{(0)}+(T-t) U_{s s}^{(1)}+(T-t)^{2} U_{s s}^{(2)}\right] \times \frac{1}{2 k^{2} s^{2 \beta}} \frac{1}{U_{x x}^{(0)}+(T-t) U_{x x}^{(1)}+(T-t)^{2} U_{x x}^{(2)}} \\
& \times\left[(\mu-r)\left(U_{x}^{(0)}+(T-t) U_{x}^{(0)}+(T-t)^{2} U_{x}^{(2)}\right)+k^{2} s^{2 \beta+1}\left(U_{x s}^{(0)}+(T-t) U_{x s}^{(1)}+(T-t)^{2} U_{x s}^{(2)}\right)\right]^{2}=0 .
\end{aligned}
$$

For convenience, we use $U$ instead of $U_{T}(x)$, that is, $U^{\prime}=U_{x}$ and $U^{\prime \prime}=U_{x x}$. Collect the different terms of $T-t$ in (12), and let the constant term equal to zero, so we can obtain the solution of $J^{(1)}(x, s)$ as follows:

$$
\begin{array}{r}
J^{(1)}(x, s)=r x U^{\prime}-\frac{(\mu-r)^{2}}{2 k^{2} s^{2 \beta}} \frac{\left(U^{\prime}\right)^{2}}{U^{\prime \prime}}, \\
\text { for all }(x, s) \in(0, \infty) \times(\infty, 0) .
\end{array}
$$

Next, we let coefficient of the term $(T-t)$ equal to 0 , and it follows that

$$
\begin{aligned}
J^{(2)}(x, s)= & \frac{1}{2 J_{x x}^{(0)}}\left[-J^{(1)} J_{x x}^{(1)}+r x\left(U_{x}^{(0)} J_{x x}^{(1)}+J_{x}^{(1)} U_{x x}^{(0)}\right)+\mu J_{s}^{(1)} U_{x x}^{(0)}+\frac{1}{2} k^{2} s^{2 \beta+2} J_{s s}^{(1)} U_{x x}^{(0)}\right. \\
& \left.-\frac{1}{2 k^{2} s^{2 \beta}}\left(2(\mu-r)^{2} U_{x}^{(0)} J_{x}^{(1)}+2(\mu-r) k^{2} s^{2 \beta+1} U_{x}^{(0)} J_{x s}^{(1)}\right)\right],
\end{aligned}
$$

where

Let

$$
\begin{aligned}
& J_{x}^{(1)}=r U^{\prime}+r x U^{\prime \prime}-\frac{(\mu-r)^{2}}{2 k^{2} s^{2 \beta}} \frac{2 U^{\prime}\left(U^{\prime \prime}\right)^{2}-\left(U^{\prime}\right)^{2} U^{\prime \prime \prime}}{\left(U^{\prime \prime}\right)^{2}}, \\
& J_{x x}^{(1)}=2 r U^{\prime \prime}+r x U^{\prime \prime \prime}-\frac{(\mu-r)^{2}}{k^{2} s^{2 \beta}}\left(U^{\prime \prime}-\frac{2 U^{\prime} U^{\prime \prime} U^{\prime \prime \prime}+\left(U^{\prime}\right)^{2} U^{\prime \prime \prime \prime}}{2\left(U^{\prime \prime}\right)^{2}}+\frac{\left(U^{\prime}\right)^{2}\left(U^{\prime \prime}\right)^{2}}{\left(U^{\prime \prime}\right)^{3}}\right), \\
& J_{s}^{(1)}=\frac{(\mu-r)^{2} \beta}{k^{2} s^{2 \beta+1}} \frac{\left(U^{\prime}\right)^{2}}{U^{\prime \prime}}, \\
& J_{s s}^{(1)}=-\frac{(\mu-r)^{2} \beta(2 \beta+1)}{k^{2} s^{2 \beta+2}} \frac{\left(U^{\prime}\right)^{2}}{U^{\prime \prime}}, \\
& J_{x s}^{(1)}=\frac{(\mu-r)^{2} \beta}{k^{2} s^{2 \beta+1}}\left(\frac{2 U^{\prime}\left(U^{\prime \prime}\right)^{2}-\left(U^{\prime}\right)^{2} U^{\prime \prime \prime}}{\left(U^{\prime \prime}\right)^{2}}\right) .
\end{aligned}
$$




$$
c_{2}:=\left(7 \max _{1 \leq i \leq 7} \sup _{\substack{x>0 \\ S \in(\infty, 0)}}\left|a_{i}\right|\right)+1 .
$$

Next, the classical subsolutions and optimal solutions $J(t, x, y)$ and $\bar{J}(t, x, y)$ of the HJB equation will be constructed, respectively. We will prove that the value function lies between the subsolution and the optimal solution, namely, $J(t, x, y) \leq J(t, x, y) \leq \bar{J}(t, x, y)$.

Define $\bar{J}_{2}:=c_{2}$ and $J_{2}:=-\bar{J}_{2}$, and let

$$
\begin{aligned}
& J:=J^{(0)}+(T-t) J^{(1)}+(T-t)^{2} J, \\
& \bar{J}:=J^{(0)}+(T-t) J^{(1)}+(T-t)^{2} \bar{J}_{2},
\end{aligned}
$$

We will prove that

$$
\begin{aligned}
& J(t, x, s) \leq J(t, x, s) \leq \bar{J}(t, x, s), \\
& \quad \text { for all }(t, x, s) \in[0, T] \times(0, \infty) \times(\infty, 0) .
\end{aligned}
$$

We consider the trading strategy $\underline{\pi}$ affected by $J(t, x, s)$ :

$$
\underline{\pi}(t, x, s)=-\frac{(\mu-r) J_{x}+k^{2} s^{2 \beta+1} J_{x s}}{k^{2} s^{2 \beta} J_{x x}} .
$$

Apply Ito's formula:

$$
\begin{aligned}
\underset{J}{J}\left(T, X_{T}^{\underline{\pi}}, S_{T}\right)-J_{-}\left(t, X_{t}^{\underline{\pi}}, S_{t}\right)= & \int_{t}^{T}\left(J_{t}+r x \underline{J}_{x}+\mu \underline{J}_{-}+\frac{1}{2} k^{2} s^{2 \beta+2} J_{s s}+(\mu-r) \underline{\pi}+\frac{1}{2} \underline{\pi}^{2} k^{2} s^{2 \beta} \underline{J}_{x x}+\underline{\pi} k^{2} s^{2 \beta+1} J_{x s}\right) \mathrm{d} u \\
& +\underbrace{\int_{t}^{T}\left(\underline{\pi} k s^{\beta} \underline{J}_{x}+k s^{\beta+1} J_{s}\right) \mathrm{d} W_{u}}_{t} .
\end{aligned}
$$

The formula is a local martingale. Define a sequence of ending time $\left\{\tau_{n}\right\}_{n=1}^{\infty}$, where $\tau_{n} \in[t, T], \tau_{n} \leq \tau_{n+1}$, $\forall n, \exists \tau_{n} \longrightarrow T$ a.s. as $n \longrightarrow \infty$. Replacing $T$ with $T \wedge \tau_{n}$, the formula of local martingale becomes

$$
\begin{aligned}
& \left.\left.\underset{-}{J}\left(T \wedge \tau_{n}, X_{T \wedge \tau_{n}}^{\pi}, S_{T \wedge \tau_{n}}\right)-J_{-}^{J}\left(t, X_{t}^{\frac{\pi}{t}}, S_{t}\right)=\int_{t}^{T \wedge \tau_{n}}(]_{t}^{J}+r x\right]_{x}+\mu s\right]_{s}+\frac{1}{2} k^{2} s^{2 \beta+2} J{ }_{s s} \\
& \left.+(\mu-r) \underline{\pi}+\frac{1}{2} \underline{\pi}^{2} k^{2} s^{2 \beta} \underline{-}_{x x}+\underline{\pi} k^{2} s^{2 \beta+1}{ }_{-x s}\right) \mathrm{d} u+\underbrace{\int_{t}^{T \wedge \tau_{n}}\left(\underline{\pi} k s^{\beta} J_{x}+k s^{\beta+1} J\right)}_{\text {local martingales }} \mathrm{d} W_{u} .
\end{aligned}
$$

Since the integrand function of the first term on the right side of equation (21) is substituted into the left side of the HJB equation of the subsolution $J$, this term is nonnegative. Taking the conditional expectations on both sides of equation (21), we have

$$
\underline{J}(t, x, y) \leq E\left[J\left(T \wedge \tau_{n}, X_{T \wedge \tau_{n}}^{\frac{\pi}{J}}, S_{T \wedge \tau_{n}}\right) \mid X_{t}^{\frac{\pi}{t}}=x, S_{t}=s\right],
$$

where $J\left(T \wedge \tau_{n}, X_{T \wedge \tau_{n}}^{\frac{\pi}{T}}, S_{T \wedge \tau_{n}}\right) \longrightarrow J\left(T, X_{t}^{\frac{\pi}{t}}, S_{t}\right)=J_{T}\left(X_{T}^{\frac{\pi}{T}}\right)$ a.s. $n \longrightarrow \infty$, so

$$
\begin{aligned}
& \left|J\left(T \wedge \tau_{n}, X_{T \wedge \tau_{n}}^{\frac{\pi}{J}}, S_{T \wedge \tau_{n}}\right)\right|=\left|J_{T}\left(X_{T \wedge \tau_{n}}^{\frac{\pi}{2}}\right)+\left(T \wedge \tau_{n}-t\right) \frac{r X_{T \wedge \tau_{n}}^{\frac{\pi}{J_{T}^{\prime}}}{ }_{T}^{\prime}\left(X_{T \wedge \tau_{n}}^{\frac{\pi}{2}}\right)-\left(1 /\left(2 k^{2} s^{2 \beta}\right)\right)(\mu-r)^{2} J_{T}^{\prime}\left(X_{T \wedge \tau_{n}}^{\frac{\pi}{2}}\right)^{2}}{J_{T}^{\prime \prime}\left(X_{T \wedge \tau_{n}}^{\frac{\pi}{\pi}}\right)}+c_{2}\left(T \wedge \tau_{n}-t\right)^{2}\right| \\
& \leq\left|J_{T}\left(X_{T \wedge \tau_{n}}^{\frac{\pi}{n}}\right)\right|+T \frac{r X_{T \wedge \tau_{n}}^{\pi}\left|J_{T}^{\prime}\left(X_{T \wedge \tau_{n}}^{\frac{\pi}{\pi}}\right)\right|-\left(1 /\left(2 k^{2} s^{2 \beta}\right)\right)(\mu-r)^{2}\left|J_{T}^{\prime}\left(X_{T \wedge \tau_{n}}^{\frac{\pi}{2}}\right)^{2}\right|}{\left|J_{T}^{\prime \prime}\left(X_{T \wedge \tau_{n}}^{\pi}\right)\right|}+c_{2} T^{2} \\
& \leq c_{3} G\left(X_{T \wedge \tau_{n}}^{\frac{\pi}{}}\right) .
\end{aligned}
$$


$\left\{G\left(X_{T \wedge \tau_{n}}^{\pi}\right)\right\}_{n=1}^{\infty}$ is an integrable random variable(see Appendix). Applying the dominance and convergence theorem, we can get the following equation:

$$
E\left[\underline{J}\left(T \wedge \tau_{n}, X_{\bar{T} \wedge \tau_{n}}^{\pi}, S_{T \wedge \tau_{n}}\right) \mid X_{t}^{\frac{\pi}{t}}=x, S_{t}=s\right] \rightarrow E\left[J_{T}\left(X_{T}^{\frac{\pi}{T}}\right) \mid X_{t}^{\frac{\pi}{t}}=x, S_{t}=s\right] \quad \text {.a.s. }
$$

When $n \longrightarrow \infty$, it follows that

$$
\underline{J}(t, x, y) \leq E\left[J_{T}\left(X_{T}^{\frac{\pi}{T}}\right) \mid X_{t}^{\frac{\pi}{2}}=x, S_{t}=s\right]
$$

According to the admissibility of $\underline{\pi}\left(t, X_{t}^{\frac{\pi}{t}}, S_{t}\right)$, $J(t, x, s) \leq J(t, x, s)$ holds.

Next, we will prove that $J(t, x, s) \leq \bar{J}(t, x, s)$, where $\bar{J}$ is the solution of the HJB equation. With the same method, we have

$$
E\left[\bar{J}\left(T \wedge \tau_{n}, X_{T \wedge \tau_{n}}^{\tilde{\pi}}, S_{T \wedge \tau_{n}}\right) \mid X_{t}^{\tilde{\pi}}=x, S_{t}=y\right] \leq \bar{J}(t, x, y),
$$

for each $n$.

From equation (23), we can prove that $\left|\bar{J}\left(T \wedge \tau_{n}, X_{T \wedge \tau_{n}}^{\tilde{\pi}}, S_{T \wedge \tau_{n}}\right)\right| \leq c_{3} G\left(X_{T \wedge \tau_{n}}^{\tilde{\pi}}\right)$, so

$$
E\left[J_{T}\left(X_{T}^{\frac{\pi}{T}}\right) \mid X_{t}^{\frac{\pi}{2}}=x, S_{t}=s\right] \leq \bar{J}(t, x, y) .
$$

We can also prove that $J(t, x, s) \leq \bar{J}(t, x, s)$. So, $J(t, x, s) \leq J(t, x, s) \leq \bar{J}(t, x, s)$. According to the definition of $\bar{J}$ and $\bar{J}$, it follows that

$$
\widehat{J}(t, x, s)=J_{T}(x)+(T-t) \frac{r x J_{T}^{\prime}(x)-\left(1 /\left(2 k^{2} s^{2 \beta}\right)\right)(\mu-r)^{2} J_{T}^{\prime}(x)^{2}}{J_{T}^{\prime \prime}(x)} .
$$

Thus, $|J(t, x, s)-\widehat{J}(t, x, s)| \leq c_{2}(T-t)^{2}$.

\section{The Asymptotic Solution}

In this paper, because there is an error between the asymptotic solution and the exact solution of the value function, the error is affected by the time $t$. In order to reduce the error, we divide $[t, T]$ into $n$ segments; the length of each segment is very small, and we calculate the approximation solution in each segment. Since the term of
$(T-t)^{2}$ is an error term, it is directly ignored when substituting into the value function to calculate the investment strategy. The asymptotic value function expression is given by

$$
\widehat{J}(t, x, s)=J^{(0)}(T, x, s)+(T-t) J^{(1)}(T, x, s) .
$$

We divide $[0, T]$ into two parts, named as the first part and the second part in turn; $\delta$ is the break point, $\delta=T / 2$. Note that the value function reaches the maximum value at $t=T$, so in the approximation process, it decreases with time from $T$ to 0 . The expression of the second part is given by

$$
\widehat{J}(t, x, s)=J^{(0)}(T, x, s)+(T-t) J^{(1)}(T, x, s), \quad \delta \leq t \leq T .
$$

Because $\widehat{J}(T, x, s)=J^{(0)}(T, x, s)=U_{T}(x)$, so we have

$$
\widehat{J}(t, x, s)=U_{T}(x)+(T-t) J^{(1)}(T, x, s), \quad \delta \leq t \leq T .
$$

Note that $t=\delta$ is the starting time of the second part and the ending time of the first part. The value of $\widehat{J}(t, x, s)$ at $t=\delta$ is given by

$$
\widehat{J}(\delta, x, s)=J^{(0)}(T, x, s)+(T-\delta) J^{(1)}(T, x, s) .
$$

The first part of the value function is given by

$\widehat{J}(t, x, s)=J^{(0)}(\delta, x, s)+(\delta-t) J^{(1)}(\delta, x, s), \quad 0 \leq t \leq \delta$.

When $t=\delta$, we have

$$
\widehat{J}(\delta, x, s)=J^{(0)}(\delta, x, s)=U_{\delta}(x) .
$$

Substituting (32) into (33), we have

$$
\begin{aligned}
\widehat{J}(t, x, s) & =\widehat{J}(\delta, x, s)+(\delta-t) J^{(1)}(\delta, x, s) \\
& =J^{(0)}(T, x, s)+(T-\delta) J^{(1)}(T, x, s)+(\delta-t) J^{(1)}(\delta, x, s), \quad t \in[0, \delta],
\end{aligned}
$$

where 


$$
\begin{aligned}
J^{(1)}(\delta, x, s)= & r x \widehat{J}_{x}(\delta, x, s)+\mu s \widehat{J}_{s}(\delta, x, s)+\frac{1}{2} k^{2} s^{2 \beta+2} \widehat{J}_{s s}(\delta, x, s) \\
& -\frac{1}{2 k^{2} s^{2 \beta}}\left[\frac{(\mu-r)^{2}\left(\widehat{J}_{x}(\delta, x, s)\right)^{2}+k^{4} s^{4 \beta+2}\left(\widehat{J}_{x s}(\delta, x, s)\right)^{2}}{\widehat{J}_{x x}(\delta, x, s)}\right. \\
& \left.+\frac{2(\mu-r) k^{2} s^{2 \beta+1} \widehat{J}_{x}(\delta, x, s) \widehat{J}_{x s}(\delta, x, s)}{\widehat{J}_{x x}(\delta, x, s)}\right] .
\end{aligned}
$$

\section{Numerical Analysis}

In this section, we compare the asymptotic solution with the analytic solution given by Gao [10] under power utility function and exponential utility function. The parameters are set as follows:

$$
r=0.03, \mu=0.12, k=16.16, \beta=-1, s=67, T=2, p=-4, q=0.05
$$

$$
\widehat{J}\left(t_{n-1}, x, s\right)=\widehat{J}\left(t_{n}, x, s\right)+\left(t_{n}-t_{n-1}\right) \widehat{J}^{(1)}\left(t_{n}, x, s\right),
$$

First, $[0, T]$ is divided into some small subintervals to satisfy $\left\{0=t_{0}<t_{1}<\cdots<t_{n-1}<t_{n}=T\right\}, n=1,2, \ldots$, so the value function $\widehat{J}\left(t_{n-1}, x, s\right)$ is

$$
\widehat{J}^{(1)}\left(t_{n}, x, s\right)=r x \widehat{J}_{x}+\mu s \widehat{J}_{s}+\frac{1}{2} k^{2} s^{2 \beta+2} \widehat{J}_{s s}-\frac{1}{2 k^{2} s^{2 \beta}}\left[\frac{(\mu-r)^{2}\left(\widehat{J}_{x}\right)^{2}}{\widehat{J}_{x x}}+\frac{k^{4} s^{4 \beta+2}\left(\widehat{J}_{x s}\right)^{2}+2(\mu-r) k^{2} s^{2 \beta+1} \widehat{J}_{x} \widehat{J}_{x s}}{\widehat{J}_{x x}}\right] \text {, }
$$

The optimal investment strategy is

$$
\widehat{\pi}_{t_{n}}^{*}=-\frac{(\mu-r) \widehat{J}_{x}+k^{2} s^{2 \beta+1} \widehat{J}_{x s}}{k^{2} s^{2 \beta} \widehat{J}_{x x}}, \quad(x, s) \in(0, \infty) \times(\infty, 0)
$$

When the interval of $[t, T]$ is divided into $n$ segments, the value function at the end time $T$ is known and recursive from the end to the beginning. $\widehat{J}(t, x, s)$ and $\widehat{\pi}_{t}^{*}$ are vectors. In the process of recursion, the end of former segment is the initial position of the behind segment. The whole process can deduce the value function of arbitrary time $t$ and the optimal strategy. According to the numerical analysis, we find the error between the asymptotic solution and analytical solution is small with both power utility function and exponential utility function.

4.1. Power Utility. Gao [10] gained the analytical solution of the value function with the power utility function. The basic form of the power utility function is $U(x)=x^{p} / p$. The value function is given by

$$
H(t, x, s)=\left(A e^{k^{-2} I(t) s^{-2 \beta}}\right)^{1-p} \frac{x^{p}}{p}
$$

where

$$
\begin{aligned}
A(t)= & e^{\left\{\lambda_{1} \beta(2 \beta+1)+(r p) /(1-p)\right)(T-t)} \\
& \left\{\frac{\lambda_{2}-\lambda_{1}}{\lambda_{2}-\lambda_{1} e^{2 \beta^{2}\left(\lambda_{1}-\lambda_{2}\right)(T-t)}}\right\}^{(2 \beta+1) /(2 \beta)}, \\
I(t)= & \frac{\lambda_{1}-\lambda_{1} e^{2 \beta^{2}\left(\lambda_{1}-\lambda_{2}\right)(T-t)}}{1-\left(\lambda_{1} / \lambda_{2}\right) e^{2 \beta^{2}\left(\lambda_{1}-\lambda_{2}\right)(T-t)}}, \\
\lambda_{1,2}= & \frac{(\mu-r p) \pm \sqrt{(1-p)\left(\mu^{2}-r^{2} p\right)}}{2 \beta(1-p)} .
\end{aligned}
$$

The optimal investment strategy is

$$
\pi_{t}=\frac{(\mu-r) x}{(1-p)\left(k s^{\beta}\right)^{2}}\left(1-\frac{2 \beta(1-p) I(t)}{\mu-r}\right) .
$$




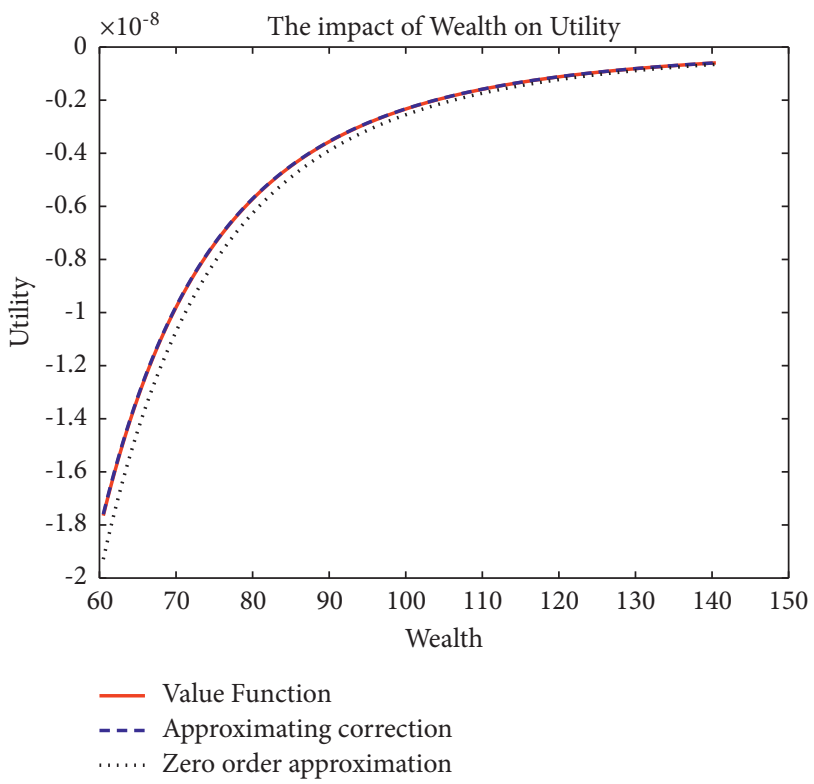

Figure 1: The impact of wealth on utility: $t=1.5$ and $T=2$.

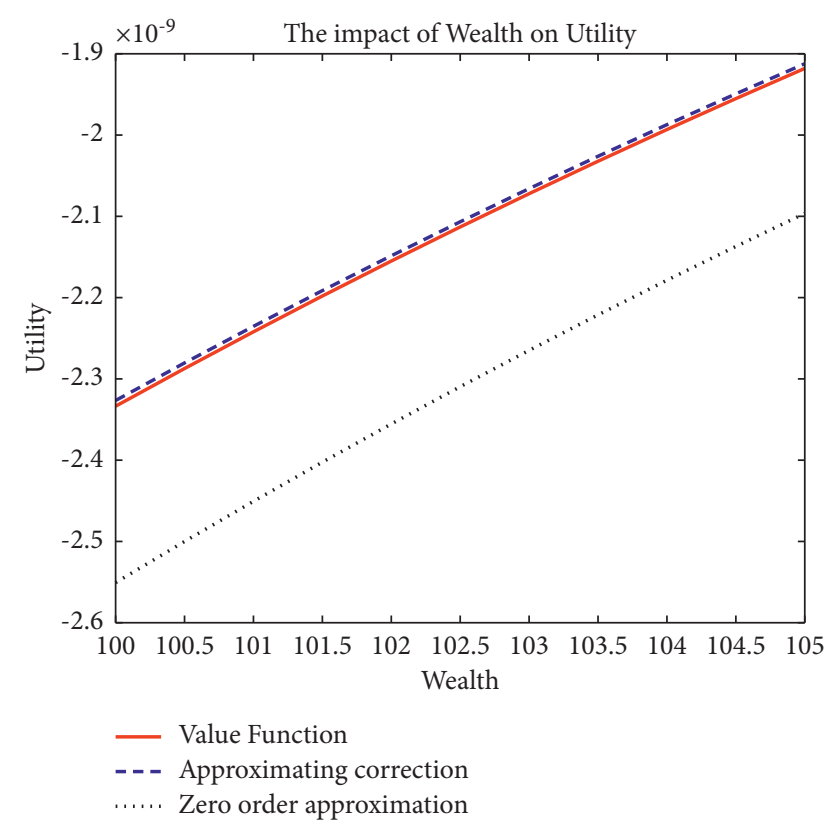

FIgURE 2: The impact of wealth on utility: $t=1.5$ and $T=2$.

Figure 1 indicates the comparison between the analytical solution and the different term of approximate solution. We find that the zero-order approximation is bigger than first-order approximation (approximation with correction).

We also find that the first-order utility asymptotic solution (approximation with correction) is very close to the analytical solution from Figures 2 and 3. The approximation error is very small when $t \longrightarrow T$.

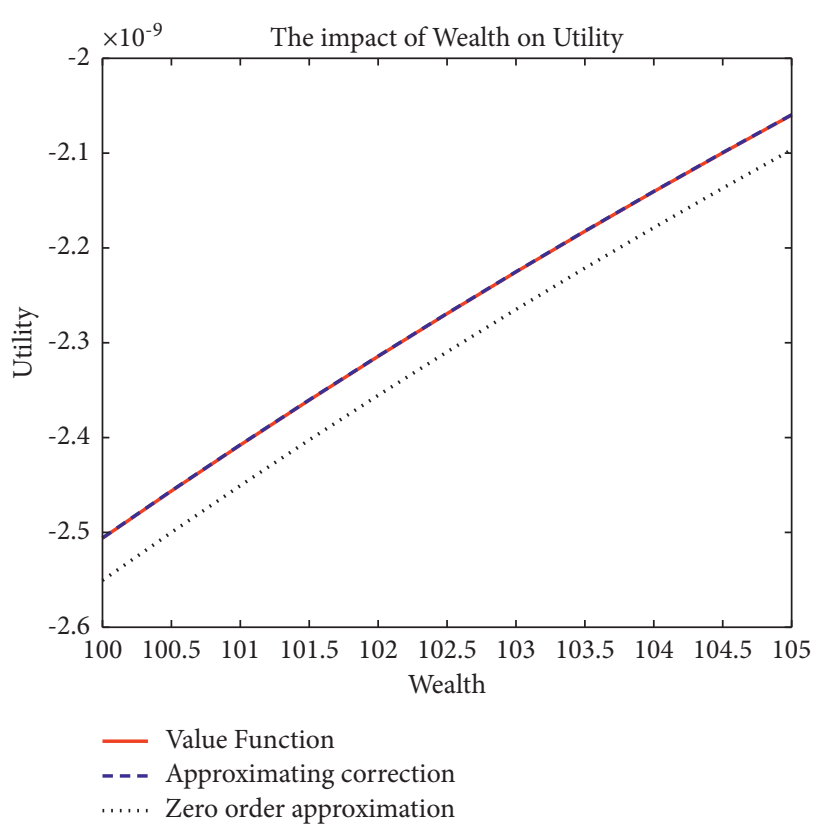

Figure 3: The impact of wealth on utility: $t=1.9$ and $T=2$.

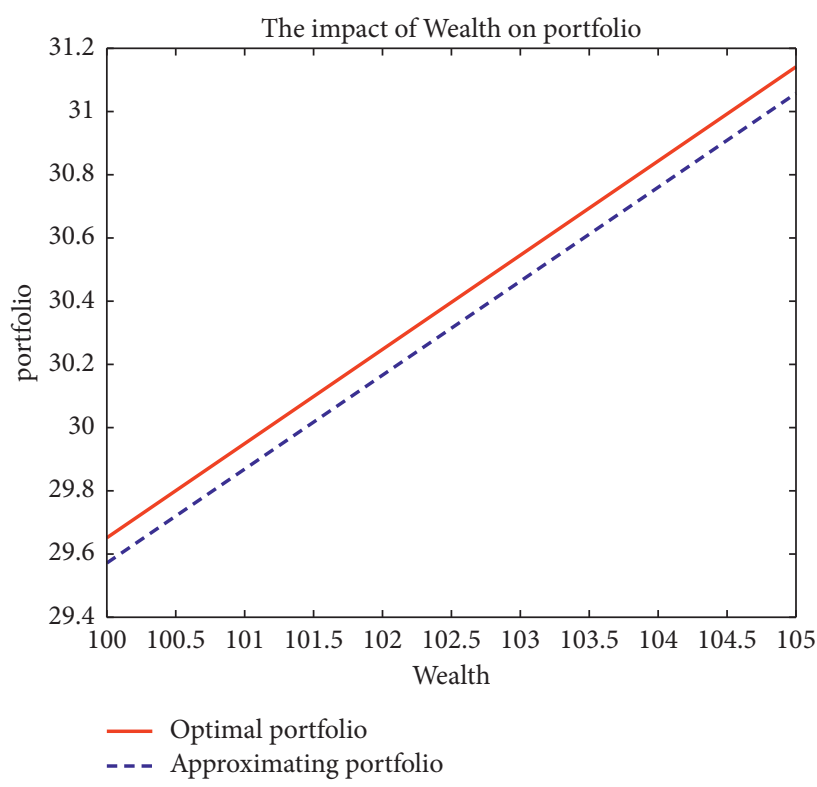

FIgURE 4: The impact of wealth on portfolio: $t=1.5$ and $T=2$.

In Figure 4 and 5, it indicates the optimal investment strategy at different time periods $t$ with first-order (approximation with correction) and zero-order asymptotic solution, respectively. We find that the error between the asymptotic solution and the analytical solution becomes smaller when the value of time $t$ is close to $T$. In order to reduce the error, a piecewise approximation is used. The result of value function is given in Figures 6 and 7 under the condition of $n=4$.

Figures 8 and 9 give the investment strategies with 4 sections. The difference is not obvious due to the large range. 


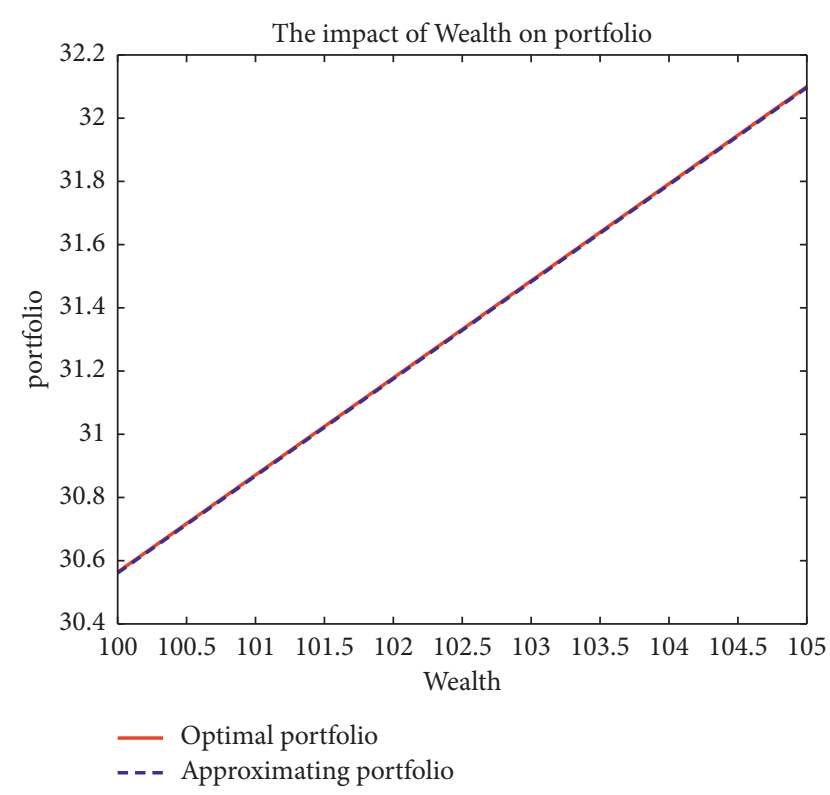

Figure 5: The impact of wealth on portfolio: $t=1.9$ and $T=2$.

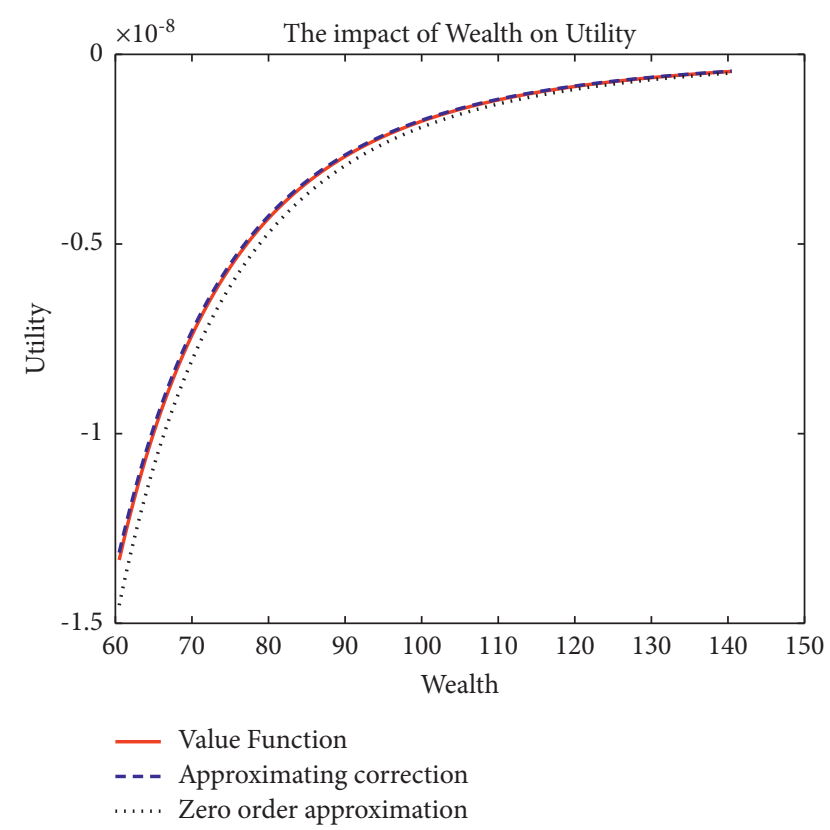

FIGURE 6: The impact of wealth on utility: $t=0, T=2$, and $n=4$.

4.2. Exponential Utility. The form of the exponential utility function is $U(x)=-(1 / q) \mathrm{e}^{-q x}$; let $\widehat{H}(t, x, s)$ represent the value function as follows:

$$
\widehat{H}(t, x, s)=-\frac{1}{q} e^{-q\left(e^{r(T-t)} x+\widehat{A}+k^{-2} I(t) s^{-2 \beta}\right)},
$$

where $\widehat{A}=\left((2 \beta+1)(\mu-r)^{2} /(4 r q)\right)\left[T-t-\left(1-e^{2 r \beta(t-T)}\right) /\right.$ $(2 r \beta)]$ and $I(t)=\left((\mu-r)^{2} / 4 r \beta q\right)\left(1-e^{2 r \beta(t-T)}\right)$.

The optimal investment strategy is given by

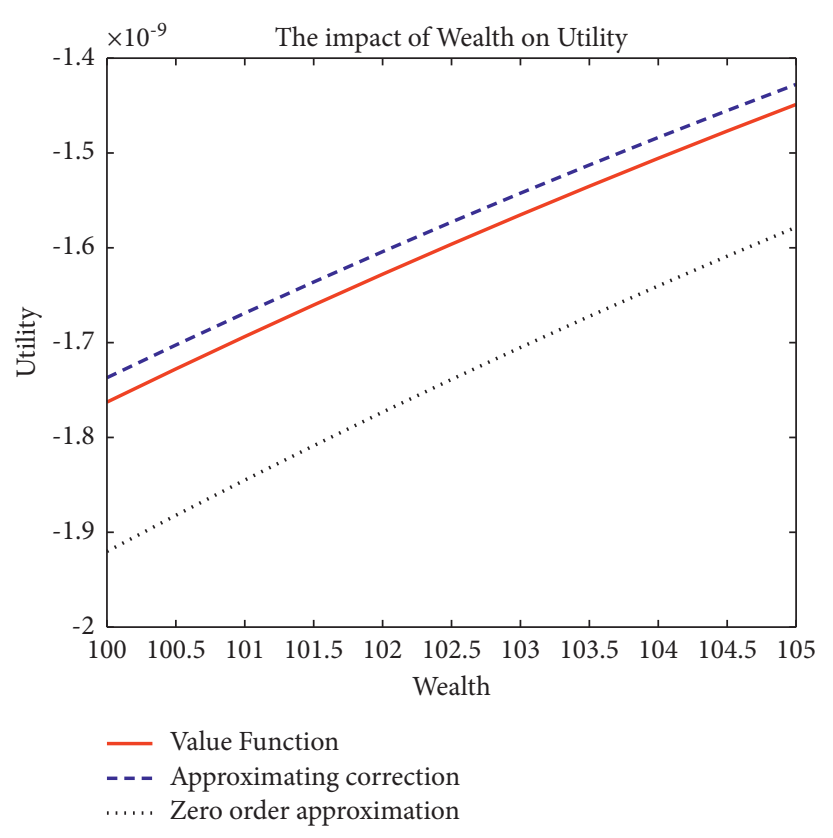

FIgURE 7: The impact of wealth on utility: $t=0, T=2$, and $n=4$.

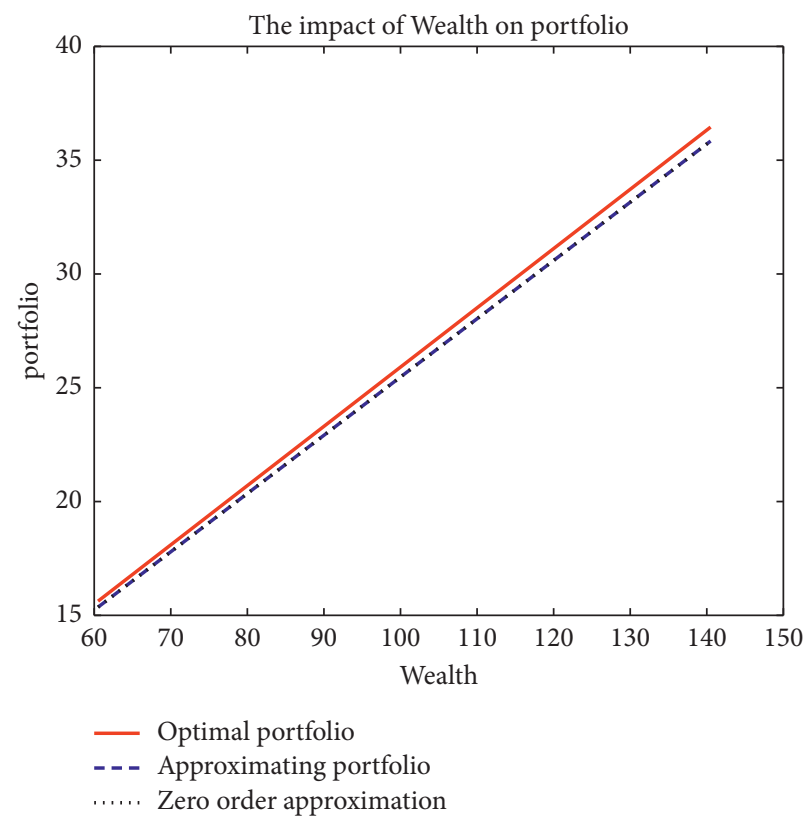

FIgURE 8: The impact of wealth on portfolio: $t=0, T=2$, and $n=4$.

$$
\widehat{\pi}_{t}=\frac{(\mu-r) e^{r(t-T)}}{q k^{2} s^{2 \beta}}\left(1+\frac{\mu-r}{2 r}\left(1-e^{2 r \beta(t-T)}\right)\right) .
$$

According to equation (44), we obtain the analytical solution and compare it with the zero-order asymptotic solution and the first-order asymptotic solution (approximation with correction), which is similar to the power utility function.

In Figure 10, the wealth $x \in[60,140]$, the zero-order approximation is $U_{T}(x)$, and the first-order approximation 


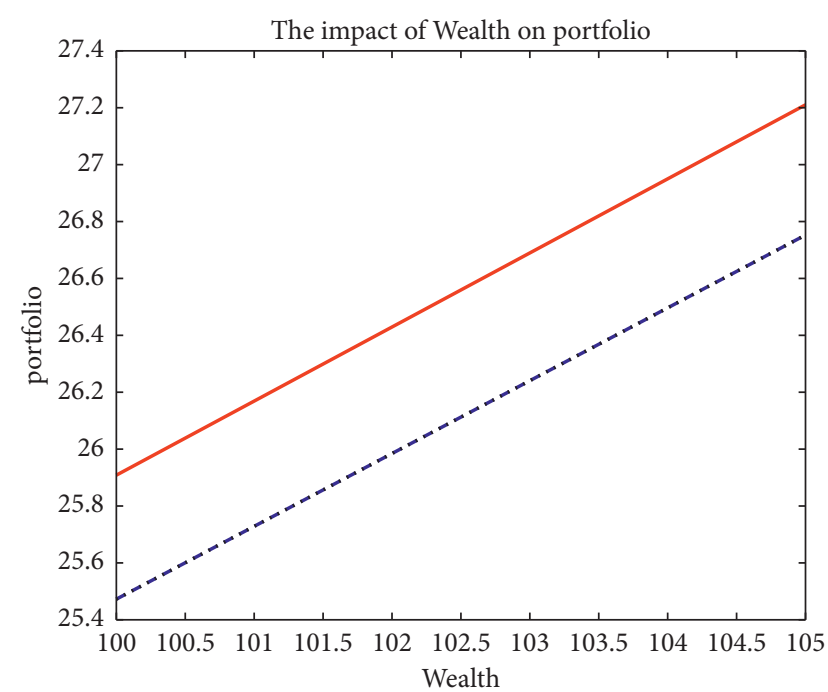

- Optimal portfolio

- - - Approximating portfolio

i.... Zero order approximation

FIgURE 9: The impact of wealth on portfolio: $t=0, T=2$, and $n=4$.

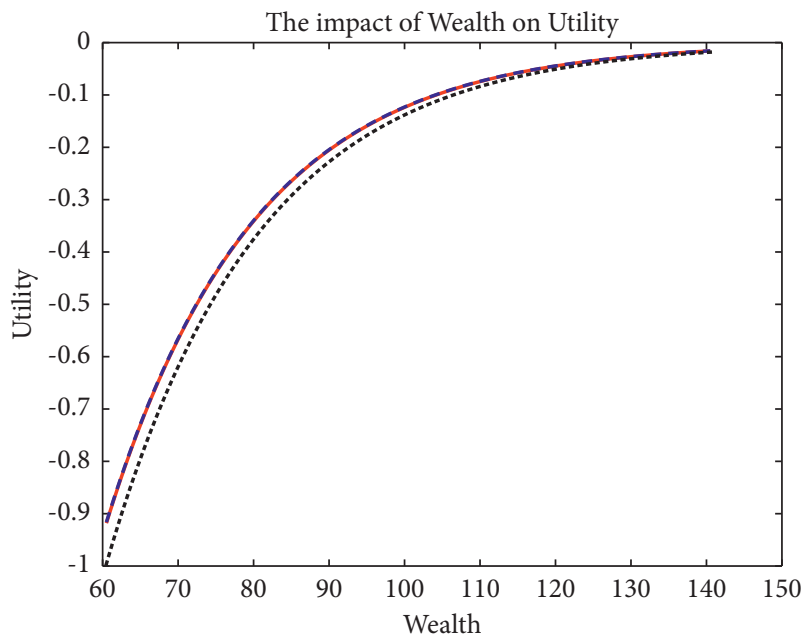

- Value Function

- - - Approximating correction

..... Zero order approximation

FIgURE 10: The impact of wealth on utility: $t=1.5$ and $T=2$.

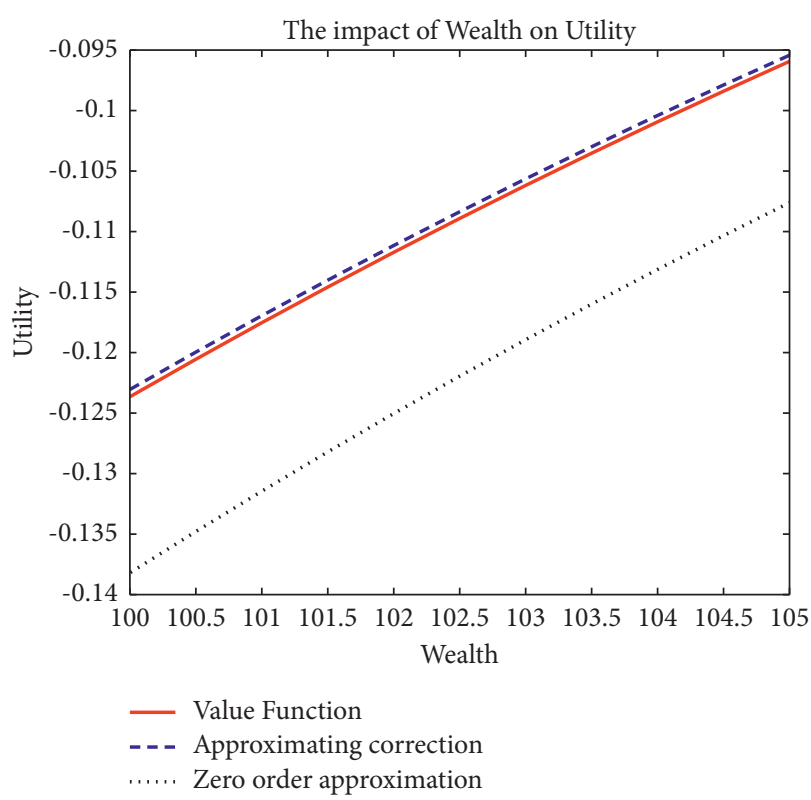

FIgURE 11: The impact of wealth on utility: $t=1.5$ and $T=2$.

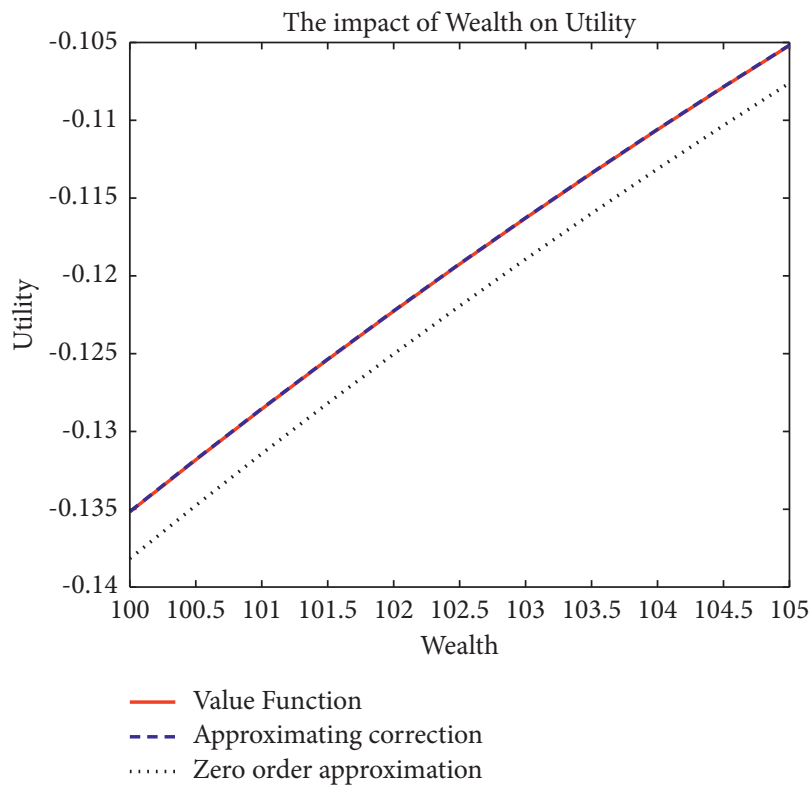

FIgURE 12: The impact of wealth on utility: $t=1.9$ and $T=2$. 


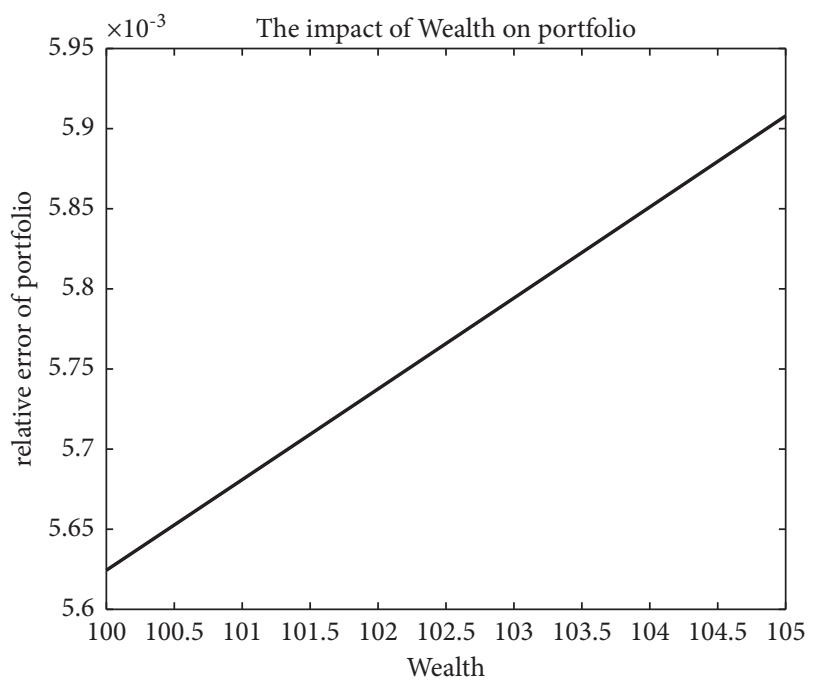

Figure 13: The impact of wealth on portfolio: $t=1.5$ and $T=2$.

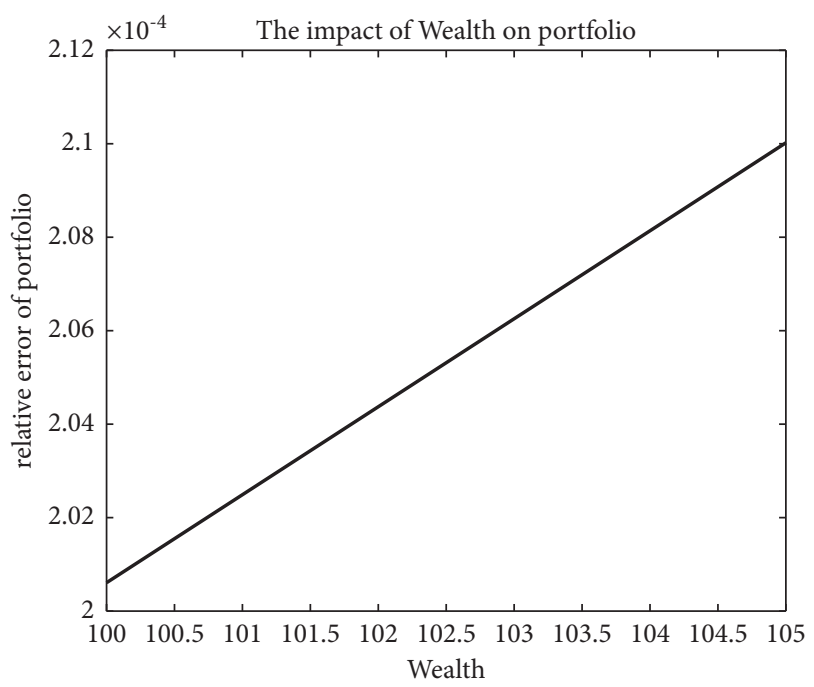

FIgURE 14: The impact of wealth on portfolio: $t=1.9$ and $T=2$.

is $\widehat{J}(x)$. We find that zero-order and the first-order approximation (approximation with correction) are very close to the analytical solution.

From Figures 11 and 12, we find the error becomes smaller. When time $t$ is closer to the terminal time $T$, it means that the asymptotic solution is better in a short time.

Figures 13 and 14 are relative errors describing investment strategies, which gradually increase with the increasing of wealth. The reason for this difference is that the analytical solution is independent of the wealth value, while the asymptotic solution is related to the wealth value. It should be noted that when the time $t$ approaches the terminal time $T$, the relative error will become smaller.

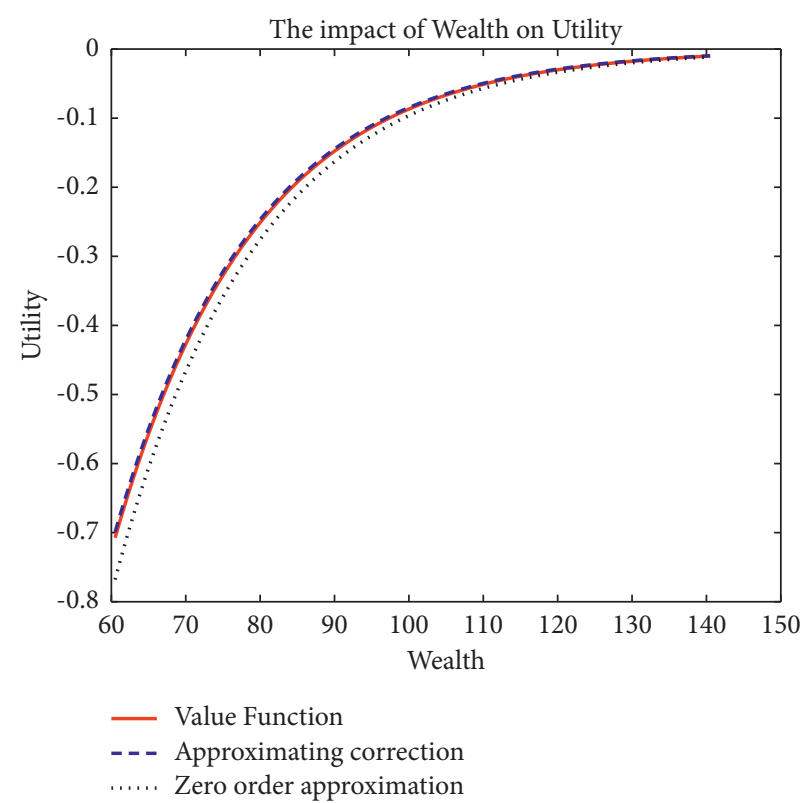

FIGURE 15: The impact of wealth on utility: $t=0, T=2$, and $n=4$.

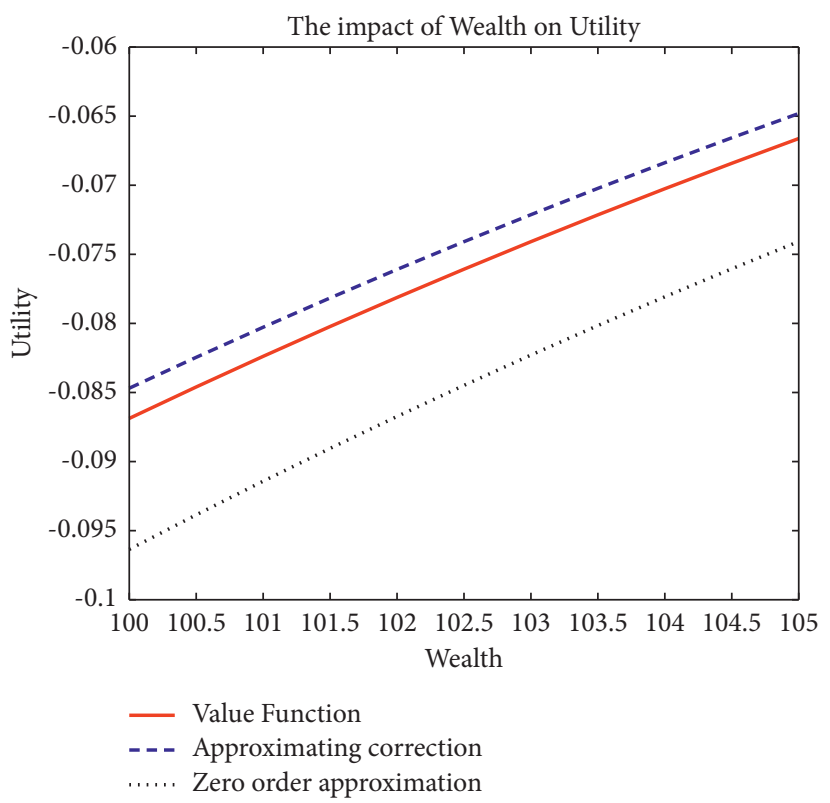

FIGURE 16: The impact of wealth on utility: $t=0, T=2$, and $n=4$.

From Figures 15 and 16, the asymptotic result is close to the analytical solution, which shows that our method is effective.

Figure 17 is the result of piecewise asymptotic analysis describing the relative error of investment strategy. When wealth increases, the relative error also increases. The reason for this difference is that the analytical solution is 


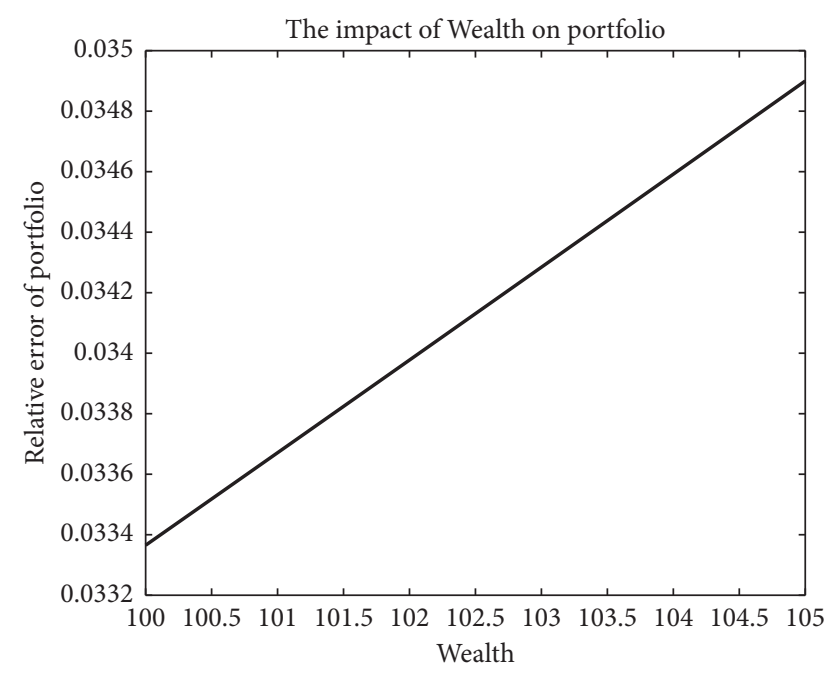

Figure 17: The impact of wealth on portfolio: $t=1.5$ and $T=2$.

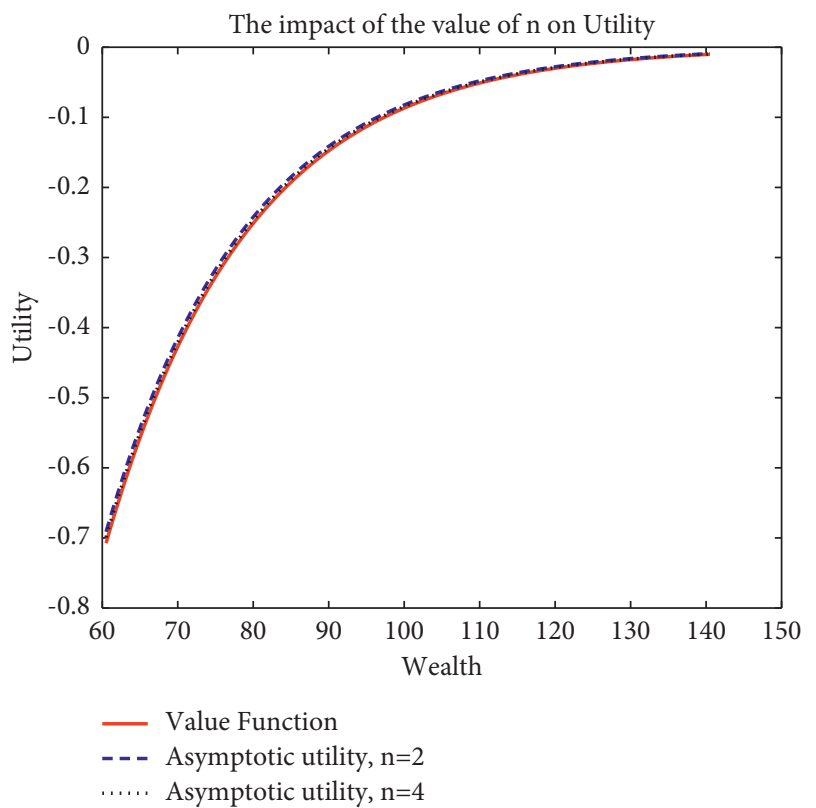

Figure 18: The impact of wealth on utility: $t=0, T=2$, and $n=4$.

independent of the wealth value, while the asymptotic solution is related to the wealth value.

In theory, $n$ can increase infinitely. In fact, we are limited by computing power. Therefore, we give the asymptotic solution of the exponential utility function in the case of $n=2$ and $n=4$ and compare it with the analytical solution.

From Figure 18, the asymptotic solution of the value function can be well fitted as $n=2$ and $n=4$. Figure 19 is a part of Figure 18, where wealth $x$ belongs to $[60,140]$. The asymptotic solution of the value function is closer to the analytical solution and converges when $n$ increases.

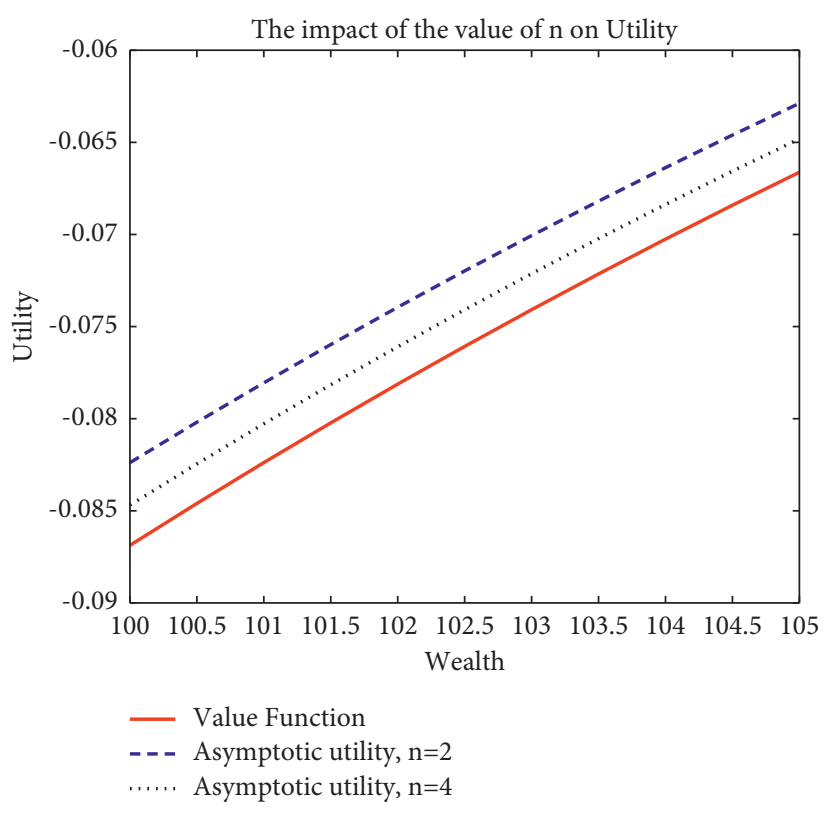

FIGURE 19: The impact of wealth on utility: $t=0, T=2$, and $n=4$.

\section{Conclusion}

This paper studies the approximation solution of HJB equation when stock price obeys the CEV model. At present, many studies have solved the exact solution of the CEV model with some special utilities, and each utility has a different exact solution, but the asymptotic solution effectively avoids this point and can be applied to general utility. From the results obtained, the asymptotic solution is significantly close to the exact solution, and the error is small, and the approximation process needs less calculation.

In the future, we can try some other methods. For examples, Gariappi [13] uses the Taylor series approximations to solve the optimal investment strategy. Ma and Zheng [11] propose an efficient dual-control Monte Carlo method to compute tight lower and upper bounds of the value function for general utilities. Our analysis can be extended to solve the investment problems in the presence of transaction costs, stochastic affine interest rates, and other uncertain factors, which will involve more complicated HJB equations to solve. We leave these work for a future study.

\section{Appendix}

Prove that $G\left(X_{T \wedge \tau_{n}}^{\pi}\right)$ is bounded. If $0<\gamma<1$, Young's inequality gives $\left(X_{T \wedge \tau_{n}}^{\pi}\right)^{1-\gamma} \leq C\left(1+\left(X_{T \wedge \tau_{n}}^{\pi}\right)^{2}\right)$. For the constant C, let $M_{v}=\int_{t}^{v} \pi_{u} k s_{u}^{\beta} X_{u}^{\pi} \mathrm{d} w_{u}$, From (3), we obtain

$$
\begin{aligned}
\left(X_{T \wedge \tau_{n}}\right)^{2}= & x^{2}+\int_{t}^{v}\left[2 X_{u}^{\pi}(\mu-r) \pi_{u}+2 r\left(X_{u}^{\pi}\right)^{2}+2 \pi_{u}^{2} k^{2} s_{u}^{2 \beta}\right] \mathrm{d} u \\
& +2 \int_{t}^{v} \pi_{u} k s_{u}^{\beta} X_{u}^{\pi} \mathrm{d} w_{u},
\end{aligned}
$$




$$
\left(X_{T \wedge \tau_{n}}\right)^{2} \leq c_{1}\left(x^{2}+2 \int_{t}^{v}\left[X_{u}^{\pi}(\mu-r) \pi_{u}+r\left(X_{u}^{\pi}\right)^{2}+\pi_{u}^{2} k^{2} s_{u}^{2 \beta}\right] \mathrm{d} u+2\left(\sup _{u \in[t, T]} M_{u}\right)^{2}\right)
$$

For the constant $c_{1}$, especially, the expected rate of return, we use Doob's maximum inequality:

$$
E\left[\left(X_{T \wedge \tau_{n}}\right)^{2}\right] \leq c_{2}\left(1+E\left[\int_{0}^{T} \pi_{u}^{2} k^{2} s_{u}^{2 t} \mathrm{~d} u\right]\right)<\infty
$$

where $c_{2}$ is a constant and $\left\{\left(X_{T \wedge \tau_{n}}^{\pi}\right)^{2}\right\}_{n=1}^{\infty}$ is a bounded random variable. So, $\left(X_{T \wedge \tau_{n}}^{\pi}\right)^{1-\gamma} \leq C\left(1+\left(X_{T \wedge \tau_{n}}^{\pi}\right)^{2}\right)$ is also a bounded random variable.

When $\gamma>1$, we apply Ito's formula to $\left(X_{v}^{\pi}\right)^{1-\gamma}$, $Z_{v}=\int_{t}^{v} \pi_{u} k s_{u}^{\beta}\left(X_{u}^{\pi}\right)^{-\gamma} \mathrm{d} w_{u}$ :

$$
\begin{aligned}
\left(X_{v}^{\pi}\right)^{1-\gamma}= & X^{1-\gamma}+(1-\gamma) \int_{t}^{v}\left[\left(X_{u}^{\pi}\right)^{-\gamma}(\mu-r) \pi_{u}+r\left(X_{u}^{\pi}\right)^{1-\gamma}-\frac{(1-\gamma) \gamma}{2} \pi_{u}^{2} k^{2} s_{u}^{2 \beta}\left(X_{u}^{\pi}\right)^{-\gamma-1}\right] \mathrm{d} u \\
& +(1-\gamma) \int_{t}^{v} \pi_{u} k s_{u}^{\beta}\left(X_{u}^{\pi}\right)^{-\gamma} \mathrm{d} w_{u} .
\end{aligned}
$$

Let $Z_{v}=\int_{t}^{v} \pi_{u} k s_{u}^{\beta}\left(X_{u}^{\pi}\right)^{-\gamma} \mathrm{d} w_{u}$, and $\pi_{u}$ is the admissible
trategy: strategy:

$$
\left(X_{v}^{\pi}\right)^{1-\gamma} \leq c_{3}\left(1+\int_{0}^{T}\left[\pi_{u}^{2} k^{2} s_{u}^{2 \beta}\left(X_{u}^{\pi}\right)^{-2 \gamma}+\pi_{u}^{2} k^{2} s_{u}^{2 \beta}\left(X_{u}^{\pi}\right)^{-2}\right] \mathrm{d} u+\left(\sup _{u \in[t, T]} Z_{u}\right)^{2}\right.
$$

For the constant $c_{3}$, use Doob's largest inequality:

$$
E\left[\left(X_{v}^{\pi}\right)^{1-\gamma}\right] \leq c_{4}\left(1+E\left[\int_{0}^{T} \pi_{u}^{2} k^{2} s_{u}^{2 \beta}\left(X_{u}^{\pi}\right)^{-2 \gamma}+\pi_{u}^{2} k^{2} s_{u}^{2 \beta}\left(X_{u}^{\pi}\right)^{-2} d u\right]+E\left(Z_{T}^{2}\right)\right.
$$

where $E\left(Z_{T}^{2}\right)=E\left[\int^{T} \pi_{u}^{2} k^{2} s_{u}^{2 \beta}\left(X_{u}^{\pi}\right)^{-2 \gamma} \mathrm{d} u\right]<\infty$; the right side of the above formala is finite.
In order to prove the result, when $G(x)=1+\log (x)$, we use the Ito formula to expand $\log \left(X_{v}^{\pi}\right)$, where $\xi_{v}=\int_{t}^{v} k s_{u}^{\beta}\left(\pi_{u} / X_{u}^{\pi}\right) \mathrm{d} w_{u}$

$$
\log \left(X_{v}^{\pi}\right)=\log x+\int_{t}^{v}(\mu-r) \frac{\pi_{u}}{X_{u}^{\pi}}+r-\frac{1}{2} k^{2} s_{u}^{2 \beta}\left(\frac{\pi_{u}}{X_{u}^{\pi}}\right)^{2} \mathrm{~d} u+\int_{t}^{v} k s_{u}^{\beta} \frac{\pi_{u}}{X_{u}^{\pi}} \mathrm{d} w_{u} .
$$

At the same time, according to Doob's largest inequality,

$$
E\left[\log \left(X_{v}^{\pi}\right)\right] \leq c_{5}\left(1+E\left[\int_{0}^{T} k^{2} s_{u}^{2 \beta}\left(\frac{\pi_{u}}{X_{u}^{\pi}}\right)^{2} d u\right]+E\left[\left(\sup _{u \in[t, T]} \xi_{u}\right)^{2}\right] .\right.
$$

$$
\log \left(X_{v}^{\pi}\right) \leq c_{5}\left(1+\int_{0}^{T} k^{2} s_{u}^{2 \beta}\left(\frac{\pi_{u}}{X_{u}^{\pi}}\right)^{2} \mathrm{~d} u+\left(\sup _{u \in[t, T]} \xi_{u}\right)^{2}\right)
$$

For the constant $c_{5}$, We hope that the expectation brings return:
Doob's inequality is given for the constant $c_{6}$ :

$$
E\left[\left(\sup _{u \in[t, T]} \xi_{u}\right)^{2}\right] \leq c_{6} E\left[\left(\xi_{T}^{2}\right)\right]=c_{6} E\left[\int_{t}^{T} k^{2} s_{u}^{2 \beta}\left(\frac{\pi_{u}}{X_{u}^{\pi}}\right)^{2} \mathrm{~d} u\right]<\infty .
$$


This shows that $\log \left(X_{v}^{\pi}\right)$ is also uniformly bounded.

\section{Data Availability}

Some or all data, models, or code that support the findings of this study are available from the corresponding author upon reasonable request.

\section{Conflicts of Interest}

The authors declare that they have no conflicts of interest.

\section{Acknowledgments}

This study was supported by Program for Chongqing Scholars and Innovative Research Team in UniversitySupport from Chongqing intelligent finance research collaborative innovation team and Natural Science Foundation of Chongqing, under Grant cstc2019jcyj-msxmx0668.

\section{References}

[1] J. C. Cox and S. A. Ross, "The valuation of options for alternative stochastic processes," Journal of Financial Economics, vol. 3, no. 1-2, pp. 145-166, 1976.

[2] J. C. Cox, "The constant elasticity of variance option pricing model," Journal of Portfolio Management, vol. 23, no. 5, pp. 15-17, 1996.

[3] D. Davydov and V. Linetsky, "Pricing and hedging pathdependent options under the CEV process," Management Science, vol. 47, no. 7, pp. 949-965, 2001.

[4] J. Detemple and W. Tian, "The valuation of American options for a class of diffusion processes," Management Science, vol. 48, no. 7, pp. 917-937, 2002.

[5] C. F. Lo, P. H. Yuen, and C. H. Hui, "Constant elasticity of variance option pricing model with time-dependent parameters," International Journal of Theoretical and Applied Finance, vol. 3, no. 4, pp. 661-674, 2000.

[6] M. Widdicks, P. W. Duck, A. D. Andricopoulos, and D. P. Newton, "The black-scholes equation revisited: asymptotic expansions and singular perturbations," Mathematical Finance, vol. 15, no. 2, pp. 373-391, 2005.

[7] K. C. Yuen, H. Yang, and K. L. Chu, "Estimation in the constant elasticity of variance model," British Actuarial Journal, vol. 7, no. 2, pp. 275-292, 2001.

[8] S. Beckers, "The constant elasticity of variance model and its implications for option pricing," The Journal of Finance, vol. 35, no. 3, pp. 661-673, 1980.

[9] D. C. Emanuel and J. D. MacBeth, "Further results on the constant elasticity of variance call option pricing model," Journal of Financial and Quantitative Analysis, vol. 17, no. 4, pp. 533-554, 1982.

[10] J. Gao, "Optimal portfolios for DC pension plans under a CEV model," Insurance: Mathematics and Economics, vol. 44, no. 3, pp. 479-490, 2009.

[11] J. Ma, W. Li, and H. Zheng, "Dual control Monte-Carlo method for tight bounds of value function in regime switching utility maximization," European Journal of Operational Research, vol. 262, no. 3, pp. 851-862, 2017.

[12] R. Kumar and H. Nasralah, "Asymptotic approximation of optimal portfolio for small time horizons," SIAM Journal on Financial Mathematics, vol. 9, no. 2, pp. 755-774, 2018.
[13] L. Gariappi and G. Skoulakis, "Taylor series approximations to expected utility and optimal portfolio choice," Mathematics and Financial Economics, vol. 5, no. 2, pp. 121-156, 2011. 